\title{
Curcumin accelerates reendothelialization and ameliorates intimal hyperplasia in balloon-injured rat carotid artery via the upregulation of endothelial cell autophagy
}

\author{
DONGDONG CHEN $^{1 *}$, XIAOYANG TAO $^{1 *}$, YANG WANG $^{1,2^{*}}$, FENGXUAN TIAN $^{1}$, YONGXIN WEI $^{1}$, \\ GUILIN CHEN $^{1}$, HAITAO SHEN ${ }^{1}$, ZHONG WANG ${ }^{1}$, ZHENGQUAN YU ${ }^{1}$, HAIYING LI $^{1}$ and GANG CHEN ${ }^{1}$ \\ ${ }^{1}$ Department of Neurosurgery and Brain and Nerve Research Laboratory, The First Affiliated Hospital of Soochow University, \\ Suzhou, Jiangsu 215006; ${ }^{2}$ Department of Neurosurgery, Anhui Provincial Hospital Affiliated to Anhui Medical \\ University, Anhui Province Key Laboratory of Brain Function and Brain Disease, Hefei, Anhui 230026, P.R. China
}

Received April 5, 2015; Accepted October 6, 2015

DOI: 10.3892/ijmm.2015.2365

\begin{abstract}
Delayed reendothelialization and intimal hyperplasia (IH) contribute to the failure of vascular interventions. Curcumin (Cur) has been used for various types of diseases with antioxidant, antiproliferative and anti-inflammatory effects. However, investigations involving the application of Cur in inhibiting IH are limited. The aim of the present study was to evaluate the potential therapeutic effects of Cur and its underlying mechanisms on a rat model of carotid artery (CA) intimal injury. In vitro, an endothelial cell (EC) migration assay was conducted using cultured primary human umbilical vein endothelial cells (HUVECs) that were exposed to Cur. In vivo, CA angioplasty injury was used to generate a rat model of intimal injury. CAs were collected at 3 days, and 1 and 4 weeks after injury, respectively, for western blot analysis and doubleimmunofluorescence analyses, terminal deoxynucleotidyl transferase-mediated dUTP nick end-labeling staining, oxidative stress indicator analysis and hematoxylin and eosin staining of the neointima. In vivo, Cur significantly enhanced the migration and healing of HUVECs and simultaneously promoted microtubule-associated protein light chain 3-II (LC3-II) expression when HUVECs were subjected to an artificial scratch. In vitro, endangium from the Cur-treated rats exhibited a significantly reduced number of apoptotic ECs and oxidative stress level compared to that of the sham group. In addition, Cur treatment markedly improved quantification
\end{abstract}

Correspondence to: Dr Gang Chen or Dr Haiying Li, Department of Neurosurgery, The First Affiliated Hospital of Soochow University, 188 Shizi Street, Suzhou, Jiangsu 215006, P.R. China

E-mail: nju_neurosurgery@163.com

E-mail: 18896581236@163.com

*Contributed equally

Key words: carotid intimal injury, intimal hyperplasia, reendothelialization, human umbilical vein endothelial cell, autophagy of the LC3-II concomitant with the downregulation of p62 in the injured CA. At 1 week following injury, sizable neointimal lesions had developed, although prominent intima thickening was not observed. At 4 weeks, apparent hemadostenosis occurred resulting from the exorbitance IH. Cur treatment markedly reduced the thickness of the neointimal lesion. It is noteworthy that high-dose Cur may have exerted more significant effects than low-dose Cur. Cur can potentially become a therapeutic drug for angiostenosis by imparting a protective effect that accelerates reendothelialization and ameliorates $\mathrm{IH}$ and was mediated by its pro-autophagic effect.

\section{Introduction}

The occurrence of angiostenosis resulting from delayed reendothelialization and intimal hyperplasia $(\mathrm{IH})$ following endovascular interventions often leads to failure of operation and remains a challenge to patients and surgeons (1). However, when the vascular integrity, particularly the endangium, is disrupted, a delay in reendothelialization and IH is inevitable. Therefore, insufficient or decelerated reendothelialization and IH are considered the two main elements that retard vascular wound healing, form atheromatous plaque and increase in-stent restenosis rates (2). However, once restenosis and thrombus have occurred, a few treatment options may be utilized and often, the therapeutic effect of these approaches becomes weak. Although the newly designed drug-eluting stents have reduced the incidence of restenosis, they are inadequate to increase reendothelialization $(3,4)$. Thus, effective drug-eluting stents with fewer side effects continue to be designed and tested for the treatment of more complex and severe arterial disease. Although vascular injury problems are the main focus of the present study, only a few interventional measures or effective agents that can promote reendothelialization and ameliorate IH after vascular injury have been developed thus far.

Curcumin (Cur), the major active component of curcuminoids, is a natural extract found in the rhizomes of turmeric of the Zingiberaceae family. Cur has long been used in China as a traditional medicine for various ailments (5). It possesses beneficial pharmacological effects, including antioxidant, 
antiproliferative (6,7), anti-inflammatory, anti-mutagenic and anti-tumorigenic properties in in vitro and in vivo models (8-10). Previous findings have shown that Cur treatment following subarachnoid hemorrhage in rat cerebrovascular disease models reduced the mortality rate and improved functional and histologic outcomes. Mounting evidence has demonstrated the potential therapeutic effect of Cur on acute ischemic stroke, possibly through mitochondrial protection, inhibition of the cell death pathway and improvement in regional cerebral blood flow (10-12). Cur also acts as an inhibitor and regulator of inflammation, restraining the lipopolysaccharide-induced overexpression of inflammatory cytokines in vascular smooth muscle cells by disrupting the TLR4-MAPK pathways and intracellular reactive oxygen species (ROS) production (13). In the peripheral vascular system, Cur may induce angiogenesis and accelerate wound healing in acute phase diabetic rats through the increased expression of vascular endothelial growth factors (14). In the cardiovascular system, the beneficial effects of Cur included reduced hypertension, improved vascular dysfunction and alleviated oxidative stress (5). Accumulating evidence has demonstrated that Cur regulates and ameliorates mechanical vascular dysfunction. However, the protective effect of Cur following vascular intimal injury, as well as the relevant mechanism associated with the ECs autophagy improvement remains to be investigated.

The abovementioned studies have demonstrated that Cur protects the nervous and vascular systems. However, the effects of Cur on accelerating reendothelialization and ameliorating IH following arterial intimal injury and the underlying mechanisms involved remain unknown. The aim of the present study was to elaborate the intimate relationship between IH and autophagy. Simultaneously, the application of Cur to an arterial intimal injury model confirmed its effects on accelerating reendothelialization and ameliorating $\mathrm{IH}$ by promoting EC autophagy.

\section{Materials and methods}

Animals. Experiments were approved by the Ethics Committee of the First Affiliated Hospital of Soochow University and were performed in accordance with the guidelines of the National Institutes of Health on the care and use of animals. Adult male Sprague-Dawley (SD) rats weighing between 270 and $300 \mathrm{~g}$ were purchased from the Animal Center of Chinese Academy of Sciences (Shanghai, China). The rats were housed in temperature- and humidity-controlled animal quarters with a 12-h light/ dark cycle. Animal body temperature was maintained at $37^{\circ} \mathrm{C}$.

Rat carotid artery balloon injury model. Subsequent to intraperitoneal anesthesia with urethane $(1,000 \mathrm{mg} / \mathrm{kg})$, an operating microscope was used to separate the carotid artery (CA). A right CA intima injury was created according to an approach described in a previous intervention project (15). Specific equipment (stereotactic head frame) was designed and manufactured by the experimental group members. In addition, a catheter and balloon were purchased from Medtronic Inc. and manufactured by the authors of this study. A T-branch pipe and 1-ml syringe were used to modulate the size and pressure of the balloon. The mode pattern is shown in Fig. 1. Briefly, the catheter was inserted through the external CA and slipped into the common CA, where it was inflated to $\sim 2 \mathrm{~atm}$ and the inner

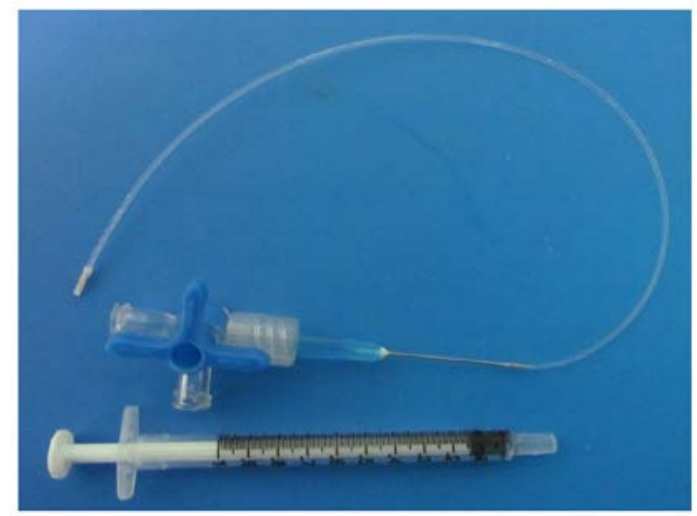

Figure 1. The instrument used in generating an intimal injury model.

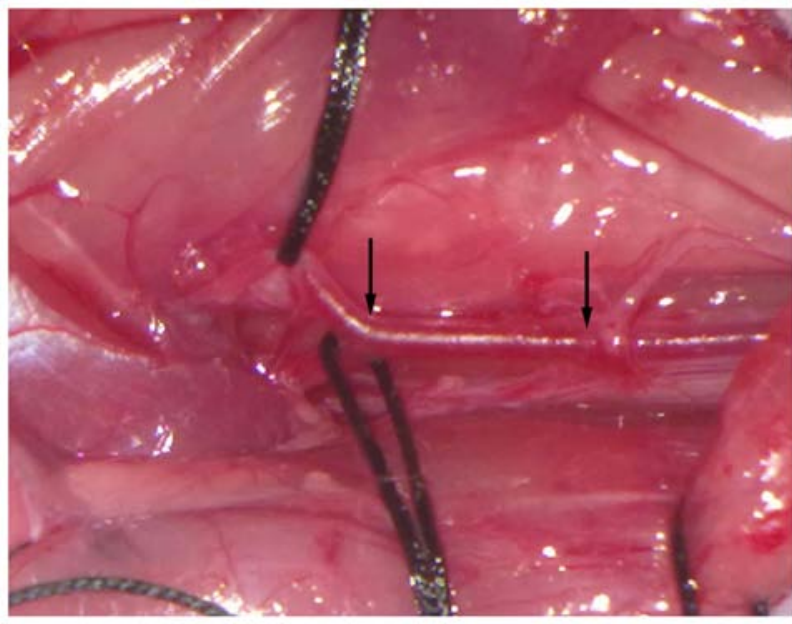

Figure 2. Photomicrograph of carotid artery intersections and the effective segment of injured carotid artery.

surface of the common CA was rubbed back and forth three times. The catheter was then removed and the impaired external CA was carefully sutured by using a 12-0 proline under the operating microscope (M651; Leica Microsystems) to prevent the impaired ECA from developing a ligature. A 2-cm common $\mathrm{CA}$ region adjacent to the common CA intersection was then cut for this experiment. The vasal site and obtained segment of the injured CA were marked by two black arrows (Fig. 2).

Drug administration and animal experiment design. The animal experiment was divided into three sections based on various time-points. The first section was used to verify that Cur was able to promote EC autophagy. A total of 90 male SD rats were randomly divided into 4 groups of 18 rats each: Sham group, injured + vehicle group, injured + Cur low-dose $(25 \mathrm{mg} / \mathrm{kg})$ group and injured + Cur high-dose $(75 \mathrm{mg} / \mathrm{kg})$ group. Cur or an equal volume of a vehicle was orally administered at $6 \mathrm{~h}$ after injury and then once daily for the subsequent 3 days. The dose of Cur administered was based on a previous study (12), thus ensuring that the animals were able to tolerate the doses without developing significant side effects. The CAs of six rats from each group were collected and homogenized in $50 \mathrm{mmol} / 1$ of ice-cold potassium phosphate buffer (PBS, pH 6.8; P1022; 


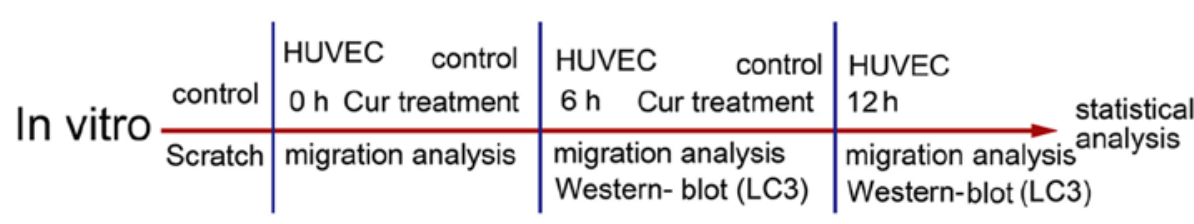

\begin{tabular}{|c|c|c|c|c|c|c|c|}
\hline & $(n=18)$ & $\begin{array}{l}\text { Sham } \\
\text { vehicle } \\
\text { Cur-L } \\
\text { Cur-H }\end{array}$ & $\begin{array}{l}72 \text { rats } \\
3 \text { days } \\
\text { CCA }\end{array}$ & $\begin{array}{r}\text { Sham } \\
\text { vehicle } \\
(n=6) \begin{array}{l}\text { Cur-L } \\
\text { Cur-H }\end{array}\end{array}$ & $\begin{array}{l}24 \text { rats } \\
1 \text { weeks } \quad(n=6) \\
\text { CCA }\end{array}$ & $\begin{array}{c}\text { Sham } \\
\text { vehicle } \\
\text { Cur-L } \\
\text { Cur-H }\end{array}$ & $\begin{array}{l}24 \text { rats } \\
4 \text { weeks } \\
\text { CCA }\end{array}$ \\
\hline & \multicolumn{2}{|c|}{$\begin{array}{c}\text { oxidative stress } \\
\text { immuno- } \\
\text { fluorescence } \\
\text { cytokines }\end{array}$} & \multicolumn{2}{|c|}{$\begin{array}{l}\text { Western- blot } \\
\text { (LC3, p62) } \\
\text { TUNEL } \\
\text { staining }\end{array}$} & \multicolumn{2}{|l|}{$\mathrm{H}$-E staining } & $\mathrm{H}-\mathrm{E}$ staining \\
\hline
\end{tabular}

Figure 3. Design of the in vitro and in vivo experiments.

Solarbio) and immediately used for the measurement of oxidative stress indicators. The CAs collected from another six rats from each group were frozen and homogenized in RIPA lysis buffer (P0013; Beyotime Institute of Biotechnology, Jiangsu, China) for western blot analysis. The CAs in the six remaining rats from each group were collected for terminal deoxynucleotidyl transferase-mediated dUTP nick end-labeling (TUNEL) staining, hematoxylin and eosin (H\&E) staining and double immunofluoresence analysis. Twenty-four rats in the second section that were housed for 2 weeks and another 24 rats housed for 4 weeks from the day of CA injury in the third section were, respectively, divided into 4 groups and their respective CAs were collected for histologic examination. The flow chart and the experimental design are shown in Fig. 3.

Cell culture and treatment. Human umbilical vein endothelial cells (HUVECs) were obtained and cultured as described previously $(16,17)$. To evaluate the effect of Cur in vitro, the cells were exposed to Cur at a concentration of $10 \mu \mathrm{M}$ for 6 and $24 \mathrm{~h}$ prior to conducting any subsequent assays (18). After the treatments, total protein of the cells was collected and stored at $-80^{\circ} \mathrm{C}$ until analysis.

Cell migration assay. The EC migration assay was performed as previously described (19). Briefly, the cells were seeded in 24-well plates. When the cells reached a post-confluent state, wounds of $1 \mathrm{~mm}$ width were created by scraping the cell monolayers with a sterile pipette tip (P3361; Seebio). The cells were treated with or without Cur, and incubated at $37^{\circ} \mathrm{C}$ for 6 and $12 \mathrm{~h}$. Migration was documented by capturing images immediately after scraping, as well as 6 and $12 \mathrm{~h}$ later at a magnification of $x 100$. Cell migration was quantified by measuring the recovered area using the NIH Image Program.

Antibodies and reagents. Cur was extracted from Curcuma longa (Bailingwei Technology Co., Ltd., Beijing, China, SB-431542). The rabbit polyclonal antibody against LC3-A/B (ab58610) and the mouse monoclonal antibody against p62 (610833) were purchased from BD Biosciences (Palo Alto, CA, USA) and the mouse monoclonal antibody against GAPDH (sc-365062) was from Santa Cruz Biotechnology, Inc. (Santa Cruz, CA, USA). The FOX3-vWF rabbit polyclonal and mouse monoclonal antibody-endothelial cell marker (ab6994 and ab68545) were from Abcam (New Territories, Shatin, Hong Kong). Secondary antibodies for western blot analysis, which included goat anti-rabbit IgG-HRP (sc-2004) and goat anti-mouse IgG-HRP (sc-2005) were purchased from Santa Cruz Biotechnology, Inc.. Normal rabbit IgG (sc-2027) was from Santa Cruz Biotechnology, Inc. Secondary antibodies for immunofluorescence, including Alexa Fluor-488 donkey anti-rabbit IgG antibody (A21206) and Alexa Fluor-555 donkey anti-mouse IgG antibody (A31570) were from Invitrogen-Life Technologies (Carlsbad, CA, USA).

Western blot analysis. Western blot analysis was performed as described previously $(20,21)$. The CAs were ground in a cell lysis buffer (P0013; Beyotime Institute of Biotechnology) using a mortar. The whole lysate was centrifuged at $200 \mathrm{x} \mathrm{g}$ for $5 \mathrm{~min}$ at $4^{\circ} \mathrm{C}$ twice. The supernatant was subsequently extracted and the protein concentration was measured using an Enhanced BCA protein assay kit (Beyotime Institute of Biotechnology). Protein samples (10 $\mu \mathrm{g} /$ lane) were loaded on a 12\% sodium dodecyl sulfate-polyacrylamide gel (P0012A; Beyotime Institute of Biotechnology), separated and electrophoretically transferred to a polyvinylidene difluoride (PVDF) membrane (Millipore Corporation, Billerica, MA, USA), which was blocked with $5 \%$ bovine serum albumin (BSA; BioSharp, Hefei, AH, China) for $1 \mathrm{~h}$ at room temperature. The membrane was then incubated overnight at $4{ }^{\circ} \mathrm{C}$ with primary antibodies. The primary antibodies were applied at a dilution of 1:1,000. The membrane was incubated with the corresponding HRP-conjugated secondary antibodies, which were diluted to 1:5,000 for $2 \mathrm{~h}$ at room temperature. The signal was developed using an enhanced chemiluminescence (ECL) kit (Beyotime Institute of Biotechnology) and exposed to $\mathrm{X}$-ray film. The films were scanned using an Epson Perfection 2480 scanner (Seiko Corp., Nagano, Japan). The relative quantity of proteins was analyzed based on the densitometric analysis using the Image $\mathrm{J}$ program and normalized to that of the loading controls. The protein levels of GAPDH served as loading controls. The densitometry ratio of the target protein to the loading control was evaluated and analyzed as the relative protein level of target protein.

Immunofluorescence microscopy. The common CA was carefully excised, fixed and embedded in paraffin, cut into $4-\mu \mathrm{m}$ 
Endothelial cells
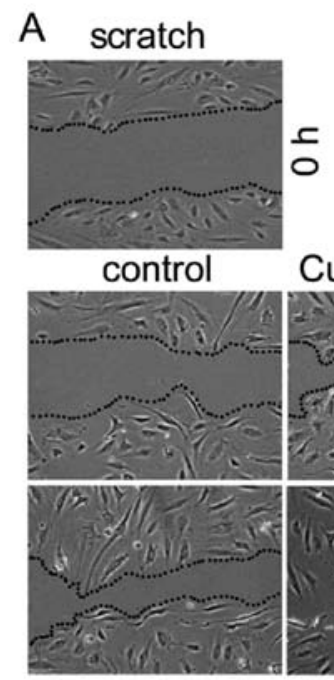

B

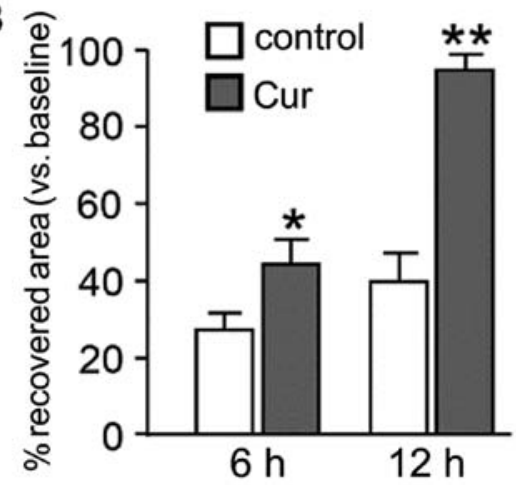

Endothelial cells

C

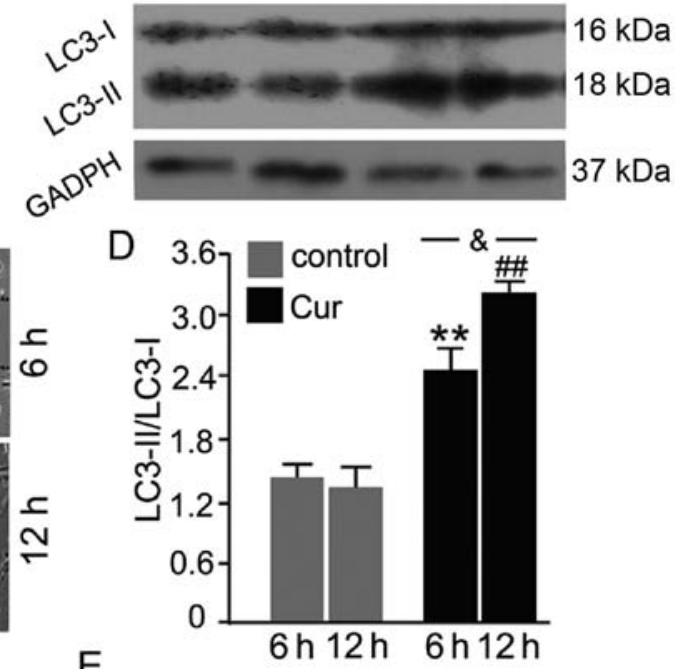

$\mathrm{E}$

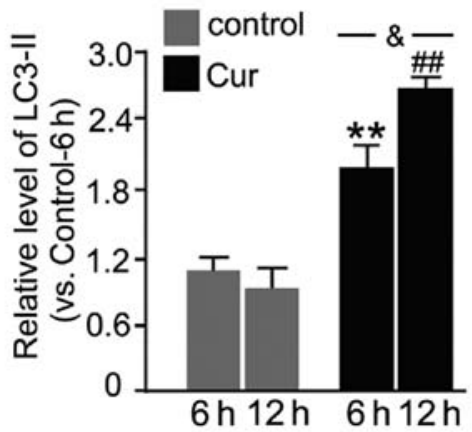

Figure 4. Effect of Cur on the migration and regeneration of HUVECs associated with autophagy. (A) Cur-enhanced closure of an artificial scratch and of HUVECs at 6 and $12 \mathrm{~h}$. Representative images are shown. (B) The recovered area was calculated when HUVECs were cultured in medium without (control) or with Cur. (C) Western blot analysis shows the microtubule-associated protein LC3 expression, and the ratio of LC-II/LC3-I in the control (6 h) , control (12 h), Cur $(6 \mathrm{~h})$, and Cur (12 h) groups. (D and E) Mean values of the protein levels of LC3-II in the control (6 h) group were normalized to 1.0. Representative images are shown. Scale bar, $200 \mu \mathrm{m}$. Data are presented as the means $\pm \mathrm{SEM} .{ }^{* *} \mathrm{P}<0.01$ vs. the control $(6 \mathrm{~h})$. ${ }^{\# \#} \mathrm{P}<0.01 \mathrm{vs}$. the control $(12 \mathrm{~h}) ;{ }^{\&} \mathrm{P}<0.05 \mathrm{vs}$. Cur $(6 \mathrm{~h})$. Cur, curcumin; HUVECs, human umbilical vein endothelial cells; LC3, light chain 3.

sections and examined by immunofluorescence staining. As previously described (20), we performed double-labeling of microtubule-associated protein light chain 3 (LC-3) and P62/von Willebrand Factor (vWF). The primary antibodies were applied at a dilution of 1:100 and the secondary antibodies were diluted to 1:300. The normal and corresponding negative controls were also included in the experiment. These methods have been previously investigated and applied by our experimental group, the fluorescence images were captured and the relative fluorescence intensity of the hybridizations was analyzed as described in our previous study (17).

Oxidative stress indicator assay. The concentrations and activitives of oxidative stress indicators in the impaired CAs were measured by using specific detection kits (Nanjing Jiancheng Bioengineering Institute, Jiangsu, China), as per the manufacturer's instructions, and as previously described (20).

TUNEL staining and quantification of apoptotic ECs. TUNEL-positive cells exhibit DNA damage, indicating apoptotic cell death (22). Apoptosis of ECs in the intimal wall was detected by TUNEL staining, following the manufacturer's protocol (DeadEnd Fluorometric kit; Promega, Madison, WI,
USA) and as described in a previous study (20). To identify vascular EC apoptosis, the sections were visualized on a fluorescence microscope. To assess the extent of EC apoptosis, six microscopic fields were examined and photographed parallel to TUNEL-positive cell counting.

$H \& E$ staining and luminal area measurement. The vessel tissue processing method was conducted and the degree of neointima formation was evaluated by measuring the luminal cross-sectional areas $(23,24)$.

Statistical analyses. Data were presented as the means \pm SEM SPSS 11.5 (SPSS Inc., Chicago, IL, USA) was used for statistical analysis. Data were analyzed by one-way ANOVA (followed by Scheffé F-test for post hoc analysis). $\mathrm{P}<0.05$ was considered statistically significant.

\section{Results}

Cur accelerated reendothelialization and migration of HUVECs. As shown in Fig. 4A and B, a comparison of recovered areas showed that Cur accelerated the closure of an artificial wound at 6 and $12 \mathrm{~h}$ after experimental induction 


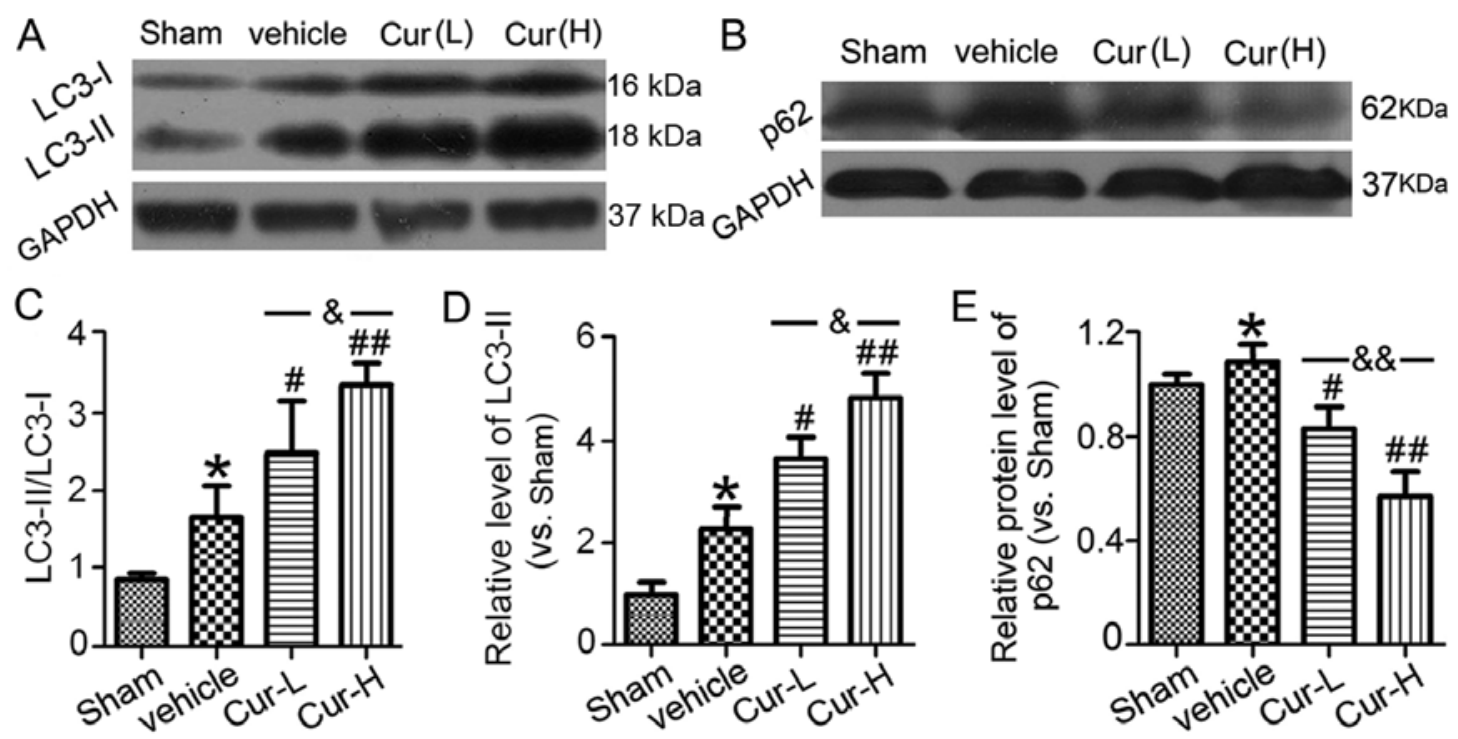

Figure 5. Cur-treated rats exhibit increased microtubule-associated protein LC3-II and reduced p62 expressions at 3 days post-treatment. (A and B) Western blot analysis detected higher protein levels of LC3-II and LC3-II/LC3-I ratios accompanied by lower protein levels of p62 in the Cur-L and Cur-H groups compared to that of the injured group. (C-E) Mean protein levels of LC3-II and p62 in the sham group were normalized to 1.0. Data are presented as the means \pm SEM. ${ }^{*} \mathrm{P}<0.05$ vs. the sham group; ${ }^{\#} \mathrm{P}<0.05$ and ${ }^{\# \#} \mathrm{P}<0.01$ vs. the vehicle group; ${ }^{\text {\& }} \mathrm{P}<0.05$ and ${ }^{\& \&} \mathrm{P}<0.05$ vs. the Cur-L group, $\mathrm{n}=6$. Cur-treated, curcumin-treated; LC3-II, light chain 3-II.

of the wound compared to the control $(\mathrm{P}<0.05$ and $\mathrm{P}<0.01$, respectively). Simultaneously, as shown in Fig. 4C-E, wherein treated control- $6 \mathrm{~h}$ was used as a standard, Cur increased the expression of LC3-II and the ratio of LC3-II/LC3-I when HUVECs were exposed to Cur, indicating that Cur induced autophagy in CAs at 6 and $12 \mathrm{~h}(\mathrm{P}<0.05$ and $\mathrm{P}<0.01$, respectively). Moreover, the expression of LC3-II and the ratio of LC3-II/LC3-I were upregulated following $12 \mathrm{~h}$ compared to that observed in the $6 \mathrm{~h}$ group $(\mathrm{P}<0.05)$.

Cur treatments enhance autophagy in vascular ECs in vitro and in vivo. To investigate the autophagy-promoting role of Cur, the autophagic levels of HUVECs from various treatment groups were evaluated by detecting LC3-II accumulation and calculating for the ratio of LC-I/ LC3-II in western blot analysis (Fig. 5). The LC3-II level and the ratio of LC3-II/LC3-I slightly increased compared to that observed in the sham group $(\mathrm{P}<0.05)$. Cur upregulated LC3-II and the ratio of LC3-II/LC3-I, indicating that Cur induced autophagy in CAs $(\mathrm{P}<0.05$ and $\mathrm{P}<0.01$, respectively). However, Cur treatment also markedly suppressed p62 expression $(\mathrm{P}<0.05$ and $\mathrm{P}<0.01$, respectively), whose level was negatively correlated with the autophagic standard. Accordingly, to distinguish the cell identity for LC3 and p62, double-immunofluorescence staining with vWF was performed on vascular sections attached to different groups (Fig. 6). A less diffuse cell staining of LC3-I was observed in the sham group, whereas a more punctate staining for LC3-II was observed in the injured group compared to that observed in the sham group. However, Cur treatment resulted in a significant increase in punctate staining compared to that observed in the sham and injured groups $(\mathrm{P}<0.01)$. An increase in p62 fluorescence intensity was detected after vascular injury, which may have been restrained by $\operatorname{Cur}(\mathrm{P}<0.01)$. Nonetheless, the high dose of Cur apparently added an extra effect to low-dose Cur $(\mathrm{P}<0.05)$.
Inhibitory effects of Cur on balloon injury-induced oxidative stress. To assess the effect of Cur on oxidative stress following CA intimal injury, we detected the levels of ROS and MDA and the activities of GSH-Px and SOD in rat CA tissues. As shown in Fig. 7, compared to the sham group, the levels of ROS $(\mathrm{P}<0.05)$ and MDA $(\mathrm{P}<0.05)$ were significantly elevated, whereas that of GSH-Px $(\mathrm{P}<0.01)$ and SOD $(\mathrm{P}<0.05)$ decreased. However, Cur treatment restrained the elevation of the levels of ROS $(\mathrm{P}<0.05)$ and MDA $(\mathrm{P}<0.05)$ and reversed the reduction of SOD $(\mathrm{P}<0.05$ and $\mathrm{P}<0.01$, respectively) and GSH-Px $(\mathrm{P}<0.05)$ activities following balloon injury. These findings showed that high-dose Cur suppressed the downregulation of SOD $(\mathrm{P}<0.05)$ and GSH-Px $(\mathrm{P}<0.05)$ activities. These results are suggestive of the antioxidant action of Cur.

Cur inhibits intimal injury-induced vascular EC apoptosis. As shown in Fig. 8A, only a few TUNEL-positive apoptotic cells were identified in the endothelium of CA in the sham group, whereas the apoptotic index was significantly higher in the injured group $(\mathrm{P}<0.01)$. Compared to the injured group, low-dosage Cur or high-dosage Cur imparted a obvious protection effect on vascular EC apoptosis $(\mathrm{P}<0.01)$.

Oral administration of Cur reverses established IH. A significant hemadostenosis or plaque was not detected in the injured and treatment groups at 1 week after injury, wherein the neointima remained fairly stable in size (Fig. 9A). The rats subjected to balloon injury of the CA after 4 weeks developed a reproducible IH response (Fig. 9A). A significant difference in the luminal area between the injured and Cur treatment groups was observed (Fig. 9B). In rats that were treated with daily Cur treatments for 4 weeks, the luminal area of CA was significantly reduced compared to that observed in the sham vessels $(\mathrm{P}<0.01)$. Cur-treated CA showed a significant suppression of IH formation compared to that observed in the injured 

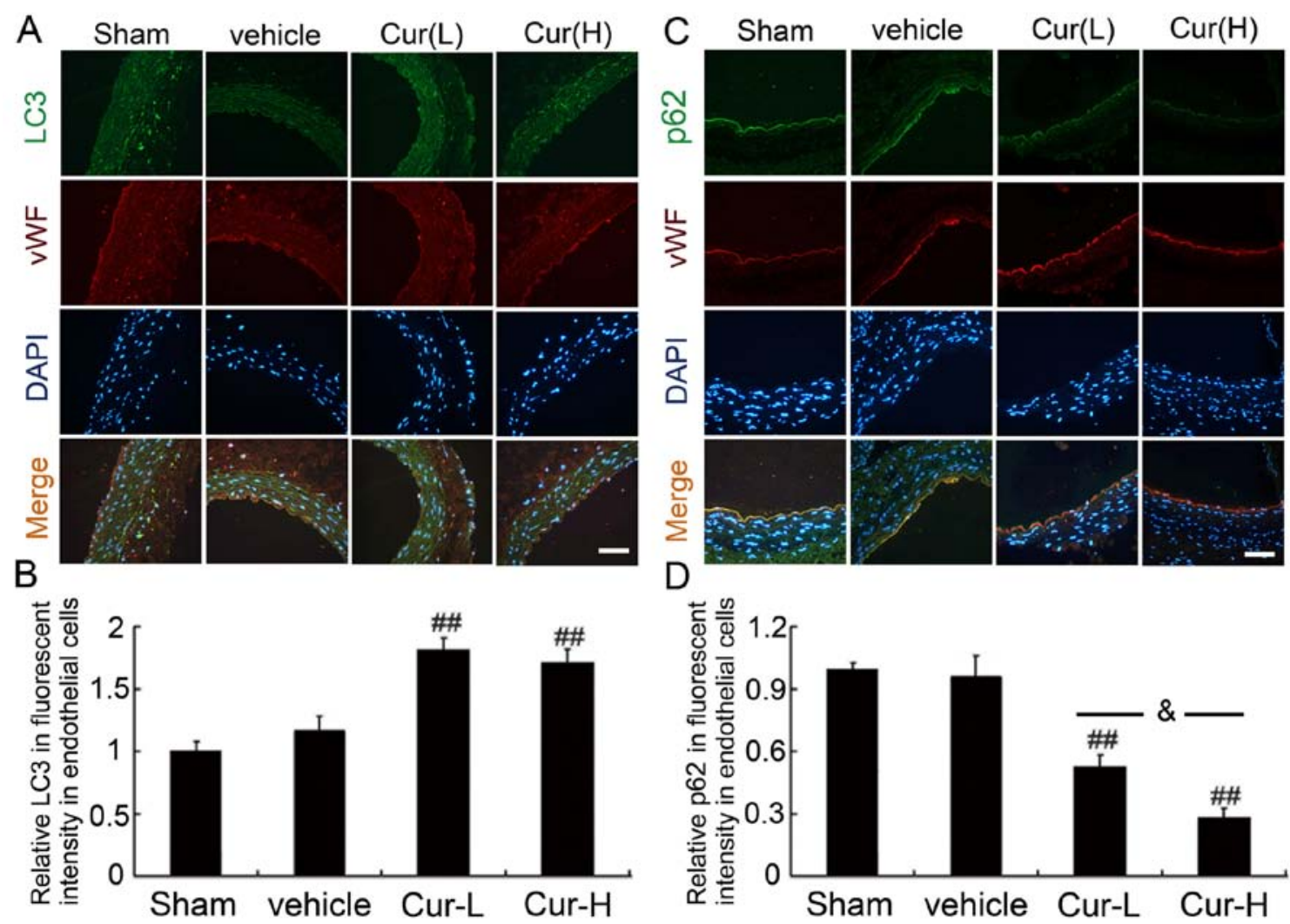

Figure 6. Cur-treated rats exhibited increased microtubule-associated protein LC3-II and reduced p62 expression confined to EC following vascular intimal injury. (A and C) Double-immunofluorescence analysis was performed with antibodies for LC3 or p62 (green) and vWF (red), and nuclei were fluorescently labeled with DAPI (blue). Bar, $80 \mu \mathrm{m}$. (B and D) The relative fluorescent intensity of LC3 and p62 in EC are shown. The mean fluorescent intensity of LC3 or p62 in ECs of the sham group was normalized to 1.0. Data are presented as the means $\pm \mathrm{SEM} .{ }^{\# \#} \mathrm{P}<0.01$ vs. the vehicle group; ${ }^{\text {\& }} \mathrm{P}<0.05$ vs. the Cur-L group, $\mathrm{n}=6$. Cur-treated, curcumin-treated; LC3-II, light chain 3-II; EC, endothelial cell; vWF, von Willebrand Factor.
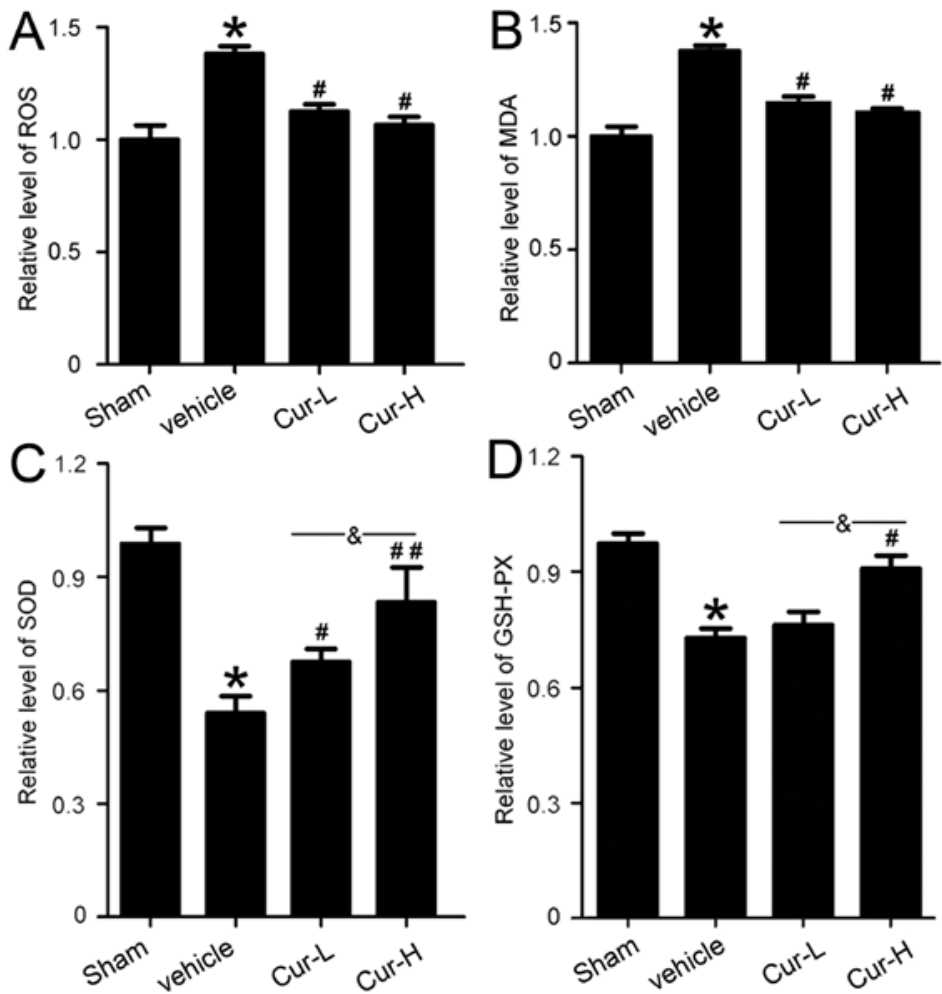

Figure 7. Cur treatment significantly reduced the level of oxidative stress following vascular intimal injury. (A-D) Quantitative analysis of the levels of ROS and MDA and the activities of SOD and GSH-Px in arterial tissues obtained at 3 days after saccule injury. The mean value of the sham group was normalized to 1.0. Data are presented as the means \pm SEM. ${ }^{*} \mathrm{P}<0.05$ vs. the sham group; ${ }^{\#} \mathrm{P}<0.05$ and ${ }^{\# \#} \mathrm{P}<0.01$ vs. the SAH group; ${ }^{\circledR} \mathrm{P}<0.05$ vs. the Cur-L group, $\mathrm{n}=6$. Cur, curcumin. 


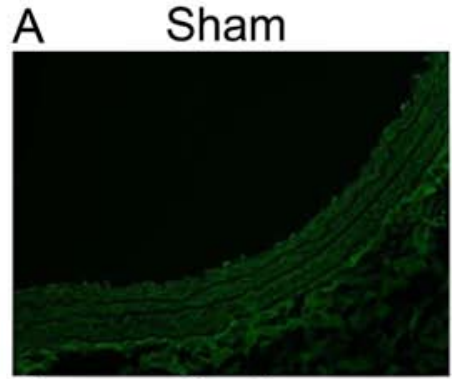

B Cur-L

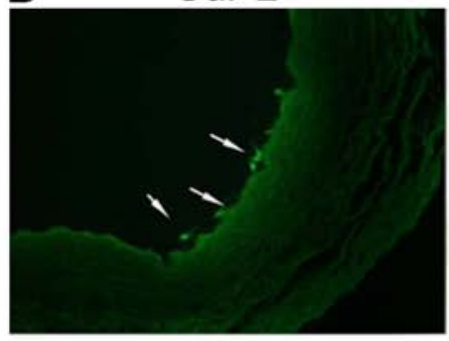

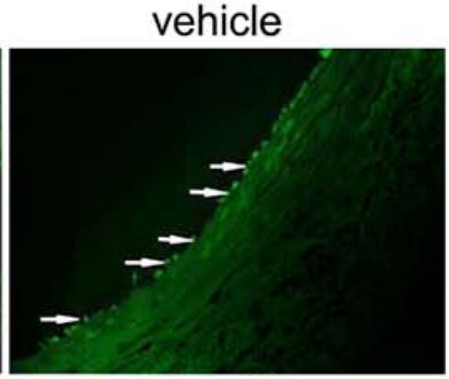

Cur-H

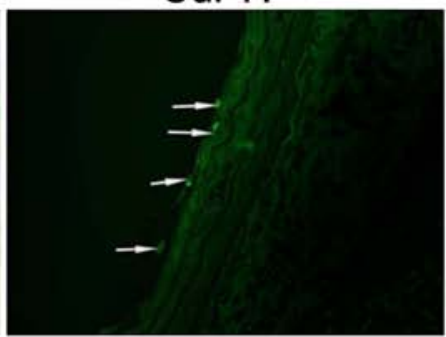

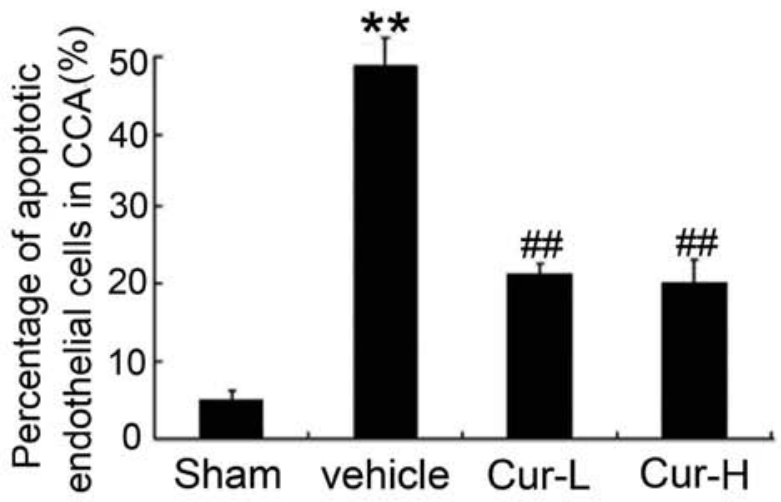

Figure 8. Cur treatment decreases EC apoptosis following vascular intimal injury. (A) Sections were labeled by terminal deoxynucleotidyl transferase-mediated dUTP nick end-labeling (TUNEL green) to detect apoptotic cells and counterstained with DAPI (blue) to detect nuclei. Arrows point to TUNEL-positive cells. Scale bar, $32 \mu \mathrm{m}$. (B) Percentage of TUNEL-positive cells. Values are presented as the means $\pm \mathrm{SEM}$. ${ }^{* *} \mathrm{P}<0.01$ vs. the sham group; ${ }^{\# \#} \mathrm{P}<0.01 \mathrm{vs}$. the vehicle group, $\mathrm{n}=6$. Cur, curcumin; EC, endothelial cell.
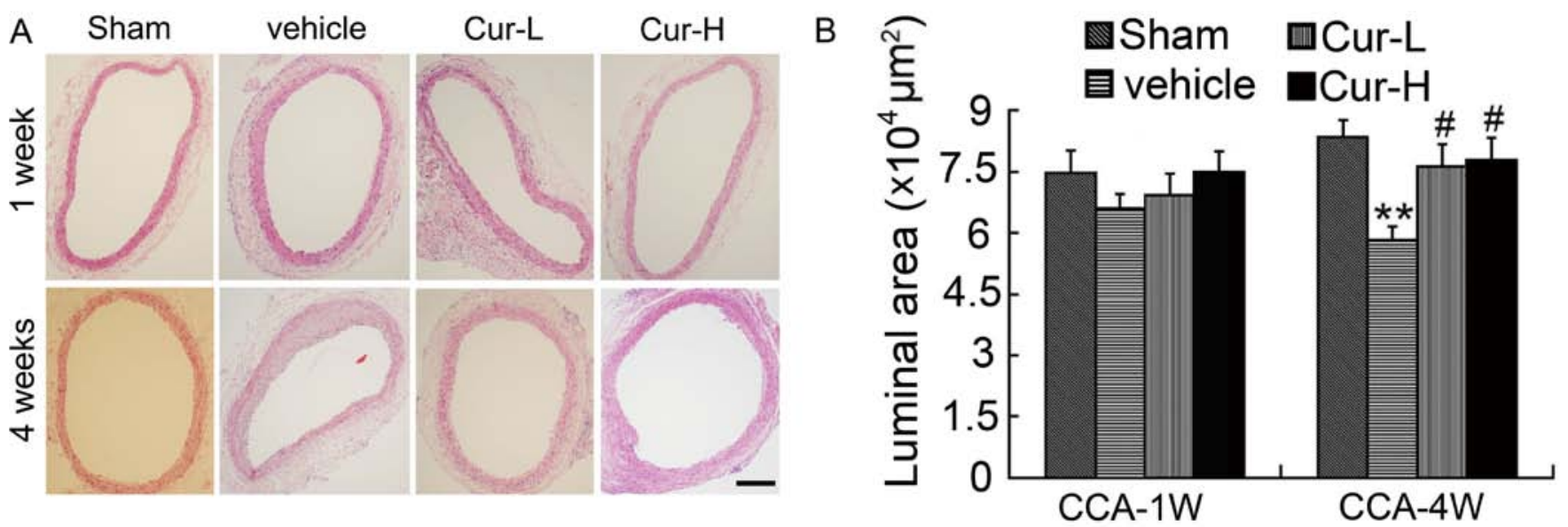

Figure 9. Curcumin (Cur) inhibits neointima formation after vascular balloon compression injury. (A) Representative images of cross-sectional areas of carotid artery (CA). Serious neointima formation was not observed at 1 week post-injury, but appeared at 4 weeks post-intimal injury, which was significantly attenuated by Cur treatment at the two doses. (B) Quantitative analysis of the average luminal area of CA from the four groups. Data are presented as the means \pm SEM. Scale bar, $100 \mu \mathrm{m}$; magnification, $\mathrm{x} 400(\mathrm{n}=6),{ }^{* *} \mathrm{P}<0.01$ vs. the sham group; ${ }^{*} \mathrm{P}<0.05$, vs. the vehicle group, $\mathrm{n}=6$.

group $(\mathrm{P}<0.05)$. However, no amelioration of neointima size and thickness was observed in the high-dose Cur group compared to that observed in the low-dose Cur group.

\section{Discussion}

Delayed reendothelialization and IH inevitably occurred on account of arterial endothelium denudation after intimal injury and played an important role in preventing in-stent thrombosis and vessel restenosis $(1,2,4)$. The results of the present study have shown that the oral administration of Cur accelerated EC migration and ameliorated $\mathrm{IH}$ in the injured $\mathrm{CA}$, which involved an increase in EC autophagy. The present results also showed that i) Cur promotes EC migration by enhancing the autophagy effect, which was confirmed by the upregulation of LC3-II and the downregulation of p62. ii) Cur markedly ameliorates IH on the injured CA after 4 weeks of induction by balloon compression, as indicated in the comparison of the extent of hemadostenosis and the expression of LC3 and p62 by western blot analysis and immunofluorescence staining.

EC dysfunction is an initial event in arterial hypertension and atherosclerosis and plays a crucial role in the regulation of CA disease $(25,26)$. In addition, the endothelium produces nitric oxide (NO) for vasorelaxation and controls leukocyte adherence, which in turn regulates vascular permeability. The Cur effects of reduced hypertension, improved vascular dysfunction and alleviated oxidative stress in mice may be 
attributable to the increase in NO bioavailability and the powerful antioxidant activity of Cur $(27,28)$. EC autophagy plays a pivotal role in regulating vascular homeostasis, and OxyLDL leads to apoptotic cell death, which is dependent on LOX-1 overexpression and the subsequent attenuation of the protective autophagic response (29).

Autophagy is associated with nutrient deprivation and a cellular mechanism of autophagy, in which proteins and organelles are encased in specialized intracellular vesicles and then broken down by lysosomal proteases for recycling. During times of stress such as nutrient starvation or treatment with certain toxic agents, the rate of autophagy is increased $(30,31)$. LC3 is a cytoplasmic protein that is rapidly cleaved to produce LC3-I and is activated by lipidation with phosphatidylethanolamine to form LC3-II by a ubiquitin-like ligation pathway. LC3-II is incorporated into the vesicle membrane and promotes membrane elongation, a variation of LC3-II that may actually be an indication of the stimulation of autophagy (32-34). $\mathrm{P} 62$, as a multidomain protein that contains different kinds of interaction domains such as the LC3-interacting region, is involved in the degradation of protein aggregates and cytoplasmic bodies via selective autophagy. Thus, p62 was used to detect autophagy flow (35). In addition, various methods have demonstrated the existence of autophagy in human atherosclerotic plaques, which may in turn contribute to the development of pharmacological approaches to stabilize vulnerable and rupture-prone lesions (36). The role of autophagy in neointima remodeling following CA mechanical injury has been previously investigated (15). However, the potential correlation between vascular ECs autophagy was not discussed intensively, although we have demonstrated the vital effect of ECs autophagy on increasing EC migration and proliferation, which coincided with the in vivo experimental results that the application of Cur, as a type of antioxidant and antiproliferative agent, accelerated reendothelialization and suppressed $\mathrm{IH}$. Thus, EC autophagy may be important in the development of neointimal lesions, where rapid cell proliferation may lead to extensive nutrient depletion.

In terms of effective intervening measures and agents, there is currently an increasing number of studies that have explored the inhibitory effect for IH. For instance, the periadventitial application of rapamycin-loaded nanoparticles resulted in the sustained inhibition of vascular restenosis, which may be used as an approach for the development of a safer, more efficacious drug delivery system for the treatment of IH (37). Local application of insulin directly onto the CA decreases neointimal thickness without affecting glycemia and apparently reduces smooth muscle cell migration after arterial injury. These results provide evidence of an insulin-coated stent that may offer protection against restenosis (38). Furthermore, delayed intermittent and repetitive inhaled carbon monoxide resulted in a significant reduction in neointima size through the inhibition of intimal and medial smooth muscle cell proliferation (15). Although Cur was equipped with various protective benefits involving the regulation of proinflammatory cytokines, growth factors, factors involved in proliferation and apoptosis, and adhesion molecules, it was applied to different animal models, including the model of atherosclerosis and cerebrovascular disease. However, relevant studies on the application of Cur in an intimal injury model have not investigated this effect (39-41).
The present study has a number of limitations. Firstly, the concrete autophagy signaling pathways remain to be adequately elucidated. Future studies should focus on the underlying formation mechanism behind the effect of Cur and analogous drugs on relieving vascular injury caused by a balloon. Secondly, use of a special inhibitor and stimulator of autophagy may facilitate in understanding the complex formation mechanism and in the development of a novel drug for the treatment of vascular intimal injury. Consequently, some drugs that target EC autophagy potentially have great therapeutic prospects for the treatment of vascular intimal injury after interventional surgery.

In conclusion, the application of Cur imparts beneficial effects by increasing the proliferation and migration of ECs by enhancing its autophagy level. Simultaneously, Cur suppresses balloon-induced IH by promoting the autophagy effect. Cur may be used as an anti-inflammatory and pro-autophagy agent and therefore serves as a novel treatment for vascular intimal injury induced by interventional therapies.

\section{Acknowledgements}

The present study was supported by grants from the National Natural Science Foundation of China (nos. 81371279, 81422013, 81471196 and 81400949), the Jiangsu Province's Outstanding Medical Academic Leader program (no. LJ201139), the Scientific Department of Jiangsu Province (no. BL2014045) and the Suzhou Government (nos. LCZX201301, SZS201413 and SYS201332) and a Project Funded by the Priority Academic Program Development of Jiangsu Higher Education Institutions.

\section{References}

1. Seedial SM, Ghosh S, Saunders RS, Suwanabol PA, Shi X, Liu B and Kent KC: Local drug delivery to prevent restenosis. J Vasc Surg 57: 1403-1414, 2013.

2. Acharjee S and Cannon CP: Duration of dual antiplatelet therapy following percutaneous coronary intervention with drug-eluting stents: A review of recent evidence. Crit Pathw Cardiol 9: 203-206, 2010.

3. Stettler C, Wandel S, Allemann S, Kastrati A, Morice MC, Schömig A, Pfisterer ME, Stone GW, Leon MB, de Lezo JS, et al: Outcomes associated with drug-eluting and bare-metal stents: A collaborative network meta-analysis. Lancet 370: 937-948, 2007.

4. Buonamici P, Marcucci R, Migliorini A, Gensini GF, Santini A, Paniccia R, Moschi G, Gori AM, Abbate R and Antoniucci D: Impact of platelet reactivity after clopidogrel administration on drug-eluting stent thrombosis. J Am Coll Cardiol 49: 2312-2317, 2007.

5. Kukongviriyapan U, Pannangpetch $\mathrm{P}$, Kukongviriyapan V Donpunha W, Sompamit K and Surawattanawan P: Curcumin protects against cadmium-induced vascular dysfunction, hypertension and tissue cadmium accumulation in mice. Nutrients 6: 1194-1208, 2014.

6. Dutta S, Padhye S, Priyadarsini KI and Newton C: Antioxidant and antiproliferative activity of curcumin semicarbazone. Bioorg Med Chem Lett 15: 2738-2744, 2005.

7. Weber WM, Hunsaker LA, Abcouwer SF, Deck LM and Vander Jagt DL: Anti-oxidant activities of curcumin and related enones. Bioorg Med Chem 13: 3811-3820, 2005.

8. Lim CS, Jin DQ, Mok H, Oh SJ, Lee JU, Hwang JK, Ha I and Han JS: Antioxidant and antiinflammatory activities of xanthorrhizol in hippocampal neurons and primary cultured microglia. J Neurosci Res 82: 831-838, 2005.

9. Biswas SK, McClure D, Jimenez LA, Megson IL and Rahman I: Curcumin induces glutathione biosynthesis and inhibits NF-kappaB activation and interleukin-8 release in alveolar epithelial cells: Mechanism of free radical scavenging activity. Antioxid Redox Signal 7: 32-41, 2005. 
10. Wang Z, Dabrosin C, Yin X, Fuster MM, Arreola A, Rathmell WK Generali D, Nagaraju GP, El-Rayes B, Ribatti D, et al: Broad targeting of angiogenesis for cancer prevention and therapy. Semin Cancer Biol: S1044-579X(15)00002-4, 2015.

11. Kuo CP, Lu CH, Wen LL, Cherng CH, Wong CS, Borel CO, Ju DT, Chen CM and Wu CT: Neuroprotective effect of curcumin in an experimental rat model of subarachnoid hemorrhage. Anesthesiology 115: 1229-1238, 2011.

12. Li S, Wu C, Zhu L, Gao J, Fang J, Li D, Fu M, Liang R, Wang L, Cheng $\mathrm{M}$, et al: By improving regional cortical blood flow, attenuating mitochondrial dysfunction and sequential apoptosis galangin acts as a potential neuroprotective agent after acute ischemic stroke. Molecules 17: 13403-13423, 2012.

13. Meng Z, Yan C, Deng Q, Gao DF and Niu XL: Curcumin inhibits LPS-induced inflammation in rat vascular smooth muscle cells in vitro via ROS-relative TLR4-MAPK/NF- $\kappa$ B pathways. Acta Pharmacol Sin 34: 901-911, 2013.

14. Kant V, Gopal A, Kumar D, Pathak NN, Ram M, Jangir BL, Tandan SK and Kumar D: Curcumin-induced angiogenesis hastens wound healing in diabetic rats. J Surg Res 193: 978-988, 2015.

15. Madigan M, Entabi F, Zuckerbraun B, Loughran P and Tzeng E: Delayed inhaled carbon monoxide mediates the regression of established neointimal lesions. J Vasc Surg 61: 1026-1033, 2014.

16. Pacifici $M$ and Peruzzi $F$ : Isolation and culture of rat embryonic neural cells: A quick protocol. J Vis $\operatorname{Exp}(63)$ : e3965, 2012.

17. Li H, Huang S, Wang S, Zhao J, Su L, Zhao B, Zhang Y, Zhang S and Miao J: Targeting annexin A7 by a small molecule suppressed the activity of phosphatidylcholine-specific phospholipase $\mathrm{C}$ in vascular endothelial cells and inhibited atherosclerosis in apolipoprotein $\mathrm{E}^{-/-}$mice. Cell Death Dis 4: e806, 2013.

18. Wang YF, Gu YT, Qin GH, Zhong L and Meng YN: Curcumin ameliorates the permeability of the blood-brain barrier during hypoxia by upregulating heme oxygenase-1 expression in brain microvascular endothelial cells. J Mol Neurosci 51: 344-351, 2013.

19. Dong Z, Cheng Y, Zhao J, Su L, Zhao B, Zhang Y, Zhang S and Miao J. Discovery of a benzoxazine derivative promoting angiogenesis in vitro and in vivo. J Cell Physiol 223: 202-208, 2010.

20. Wang Y, Gao A, Xu X, Dang B, You W, Li H, Yu Z and Chen G The Neuroprotection of Lysosomotropic Agents in Experimental Subarachnoid Hemorrhage Probably Involving the Apoptosis Pathway Triggering by Cathepsins via Chelating Intralysosomal Iron. Mol Neurobiol (Aug): 12, 2014.

21. Li H, Gao A, Feng D, Wang Y, Zhang L, Cui Y, Li B, Wang Z and Chen G: Evaluation of the protective potential of brain microvascular endothelial cell autophagy on blood-brain barrier integrity during experimental cerebral ischemia-reperfusion injury. Transl Stroke Res 5: 618-626, 2014.

22. Schlunk F, Schulz E, Lauer A, Yigitkanli K, Pfeilschifter W, Steinmetz H, Lo EH and Foerch C: Warfarin pretreatment reduces cell death and MMP-9 activity in experimental intracerebral hemorrhage. Transl Stroke Res 6: 133-139, 2015.

23. Wang Z, Chen G, Zhu WW, Bian JY, Shen XO and Zhou D: Influence of simvastatin on microthrombosis in the brain after subarachnoid hemorrhage in rats: A preliminary study. Ann Clin Lab Sci 40: 32-42, 2010.

24. Zhang JM, Wang Y, Miao YJ, Zhang Y, Wu YN, Jia LX, Qi YF and Du J: Knockout of CD8 delays reendothelialization and accelerates neointima formation in injured arteries of mouse via TNF- $\alpha$ inhibiting the endothelial cells migration. PLoS One 8 : e62001, 2013.
25. Sitia S, Tomasoni L, Atzeni F, Ambrosio G, Cordiano C, Catapano A, Tramontana S, Perticone F, Naccarato P, Camici P, et al: From endothelial dysfunction to atherosclerosis. Autoimmun Rev 9: 830-834, 2010

26. Andoh J, Sawyer B, Szewczyk K, Nortley M, Rossetti T, Loftus IM, Yáñez-Muñoz RJ and Hainsworth AH: Transgene delivery to endothelial cultures derived from porcine carotid artery ex vivo. Transl Stroke Res 4: 507-514, 2013.

27. Tesfamariam B and DeFelice AF: Endothelial injury in the initiation and progression of vascular disorders. Vascul Pharmacol 46: 229-237, 2007.

28. Lindner V, Fingerle $\mathrm{J}$ and Reidy MA: Mouse model of arterial injury. Circ Res 73: 792-796, 1993.

29. Mollace V, Gliozzi M, Musolino V, Carresi C, Muscoli S, M 1 - 145 ollace R, Tavernese A, Gratteri S, Palma E, Morabito C, et al: Oxidized LDL attenuates protective autophagy and induces apoptotic cell death of endothelial cells: Role of oxidative stress and LOX-1 receptor expression. Int J Cardiol 184: 152-158, 2015.

30. Lalaoui N, Lindqvist LM, Sandow JJ and Ekert PG: The molecular relationships between apoptosis, autophagy and necroptosis. Semin Cell Dev Biol 39: 63-69, 2015.

31. Luo T, Park Y, Sun X, Liu C and Hu B: Protein misfolding, aggregation, and autophagy after brain ischemia. Transl Stroke Res 4: 581-588, 2013.

32. Matsui Y, Takagi H, Qu X, Abdellatif M, Sakoda H, Asano T, Levine B and Sadoshima J: Distinct roles of autophagy in the heart during ischemia and reperfusion: Roles of AMP-activated protein kinase and Beclin 1 in mediating autophagy. Circ Res 100: 914-922, 2007.

33. Mizushima N and Yoshimori T: How to interpret LC3 immunoblotting. Autophagy 3: 542-545, 2007.

34. Martinet W and De Meyer GR: Autophagy in atherosclerosis: A cell survival and death phenomenon with therapeutic potential. Circ Res 104: 304-317, 2009.

35. Lin X, Li S, Zhao Y, Ma X, Zhang K, He X and Wang Z: Interaction domains of p62: A bridge between p62 and selective autophagy. DNA Cell Biol 32: 220-227, 2013.

36. Liu H, Cao Y, Tong T, Shi J, Zhang Y, Yang Y and Liu C: Autophagy in atherosclerosis: A phenomenon found in human carotid atherosclerotic plaques. Chin Med J (Engl) 128: 69-74, 2015.

37. Shi X, Chen G, Guo LW, Si Y, Zhu M, Pilla S, Liu B, Gong S and Kent KC: Periadventitial application of rapamycin-loaded nanoparticles produces sustained inhibition of vascular restenosis. PLoS One 9: e89227, 2014

38. Chiang S, Breen DM, Guo J, Mori Y and Giacca A: Local insulin application on the carotid artery inhibits neointima formation. Can J Physiol Pharmacol 91: 1086-1094, 2013.

39. Masuda T, Hidaka K, Shinohara A, Maekawa T, Takeda Y and Yamaguchi $\mathrm{H}$ : Chemical studies on antioxidant mechanism of curcuminoid: Analysis of radical reaction products from curcumin. J Agric Food Chem 47: 71-77, 1999.

40. Sikora E, Scapagnini G and Barbagallo M: Curcumin, inflammation, ageing and age-related diseases. Immun Ageing 7: 1, 2010.

41. Lapchak PA and McKim JM Jr: CeeTox ${ }^{\mathrm{TM}}$ Analysis of CNB-001 a Novel Curcumin-Based Neurotrophic/Neuroprotective Lead Compound to Treat Stroke: Comparison with NXY-059 and Radicut. Transl Stroke Res 2: 51-59, 2011. 